\title{
Measurement and Analysis of Link Quality in Wireless Networks: An Application Perspective
}

\author{
Vinay Kolar* Saquib Razak* \\ * Department of Computer Science, \\ Carnegie Mellon University, Qatar. \\ \{vkolar,srazak\}@qatar.cmu.edu
}

\author{
Petri Mähönen ${ }^{\dagger}$ \\ $\dagger$ Department of Wireless Networks, \\ RWTH Aachen University, Germany. \\ pma@mobnets.rwth-aachen.de
}

\author{
Nael B. Abu-Ghazaleh ${ }^{\ddagger}$ \\ $\ddagger$ Department of Computer Science, \\ State University of New York, Binghamton. \\ nael@cs.binghamton.edu
}

\begin{abstract}
Estimating the quality of wireless link is vital to optimize several protocols and applications in wireless networks. In realistic wireless networks, link quality is generally predicted by measuring received signal strength and error rates. Understanding the temporal properties of these parameters is essential for the measured values to be representative, and for accurate prediction of performance of the system. In this paper, we analyze the received signal strength and error rates in an IEEE 802.11 indoor wireless mesh network, with special focus to understand its utility to measurement based protocols. We show that statistical distribution and memory properties vary across different links, but are predictable. Our experimental measurements also show that, due to the effect of fading, the packet error rates do not always monotonically decrease as the transmission rate is reduced. This has serious implications on many measurementbased protocols such as rate-adaptation algorithms. Finally, we describe real-time measurement framework that enables several applications on wireless testbed, and discuss the results from example applications that utilize measurement of signal strength and error rates.
\end{abstract}

\section{INTRODUCTION}

In wireless networks, several applications and protocols utilize link quality estimates to enhance the performance of the system. However, precise characterization of wireless links in realistic wireless networks is a challenging problem since the links experience frequent channel variations and complex interference patterns. Simulators and analytical models abstract wireless propagation and interference effects to predict link quality. However, such link quality estimation techniques cannot be directly employed in realistic networks since wireless environment changes dynamically. Even if the changes are relatively slower, link quality estimation requires detailed data about wireless environment and lower level physical layer information such as exact terrain information and coherence time of the channel. Such data is generally inaccessible or even unavailable - to the higher layer protocols. Hence, estimating the link quality is an important and challenging problem in realistic operational wireless networks.

In realistic wireless networks, models [1], [2] and protocols [3], [4] adopt a more direct and approximate approach to estimate the link quality. They measure the accessible parameters that approximate link quality, and use them for modeling or optimizing the system. We henceforth refer to such measurement based models, protocols and applications by a common term: Measurement Based Applications (MBAs).
Examples of MBAs in wireless networks include AP association, transmission rate-control and routing. Existing studies have shown that MBAs are more resilient to dynamic changes in network topology and traffic, and outperform the non-measurement based counterparts by a large margin [3], [4]. MBAs capture the effectiveness of the wireless link by measuring important PHY and MAC level parameters that are provided by wireless cards.

In a large number of MBAs, measured values of Received Signal Strength (RSS) and error rates are used as decision parameters to optimize the system. RSS of a packet is directly accessible from IEEE 802.11 wireless cards, which are used on most commercial wireless networks such as WLAN and mesh network. Packet/bit error rates (PER/BER) are measured, generally by introducing additional measurement packets. While current MBAs utilize these values to predict or improve network performance, existing studies do not analyze characteristics of RSS and error rates that are directly relevant to the MBAs. There is a general discord about the temporal variation and distributions of RSS and PER which have not been thoroughly validated by experiments. While some research work assume these parameters as constants [5], [6], others approximate them as a sequence of independent and identically-distributed (i.i.d.) random variables from some known statistical distribution [1], [7].

The focus of the paper is to study the characteristics of RSS and PER with specific focus to MBAs and to develop a real-time measurement based framework that enables efficient MBA development. Our first contribution is to empirically study the statistical properties of RSS and PER. We validate the assumptions about RSS and PER by analyzing the independence and distribution of RSS and error rates for different links. We show that the distribution of the RSS and PER values are strongly dependent on the error rate of the link. Strong and weak links are separated by a layer of transitional gray zone where fading of the channel leads to unexpected and interesting distribution of the error rates. We analyze the variation of error rates with respect to transmission rates. Contrary to the commonly accepted convention, our traces indicate that error rates do not always decrease as we switch to a lower transmission rate. In fact, in a some of the cases, switching to higher transmission rate leads to a more stable link with lower error rate in the transitional gray zone. The 


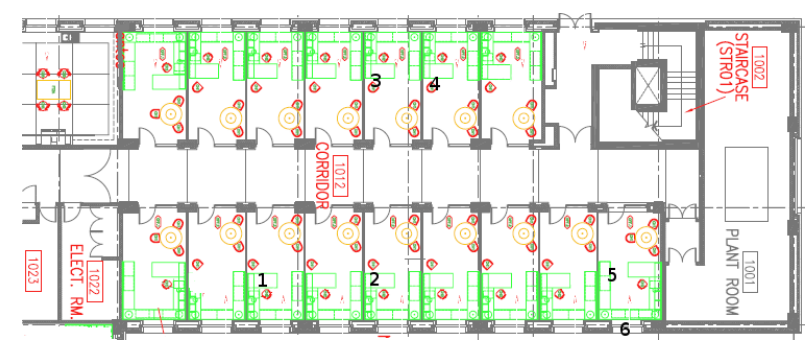

Fig. 1. Testbed

above analysis and conclusions have significant implications on MBAs. We discuss the statistical analysis in Section II.

The next contribution of the paper is a real-time measurement framework that constantly monitors the link quality. Section III describes our testbed, system architecture and measurement modules that facilitate statistical monitoring of link quality. We then briefly discuss example MBAs that utilize the measurement framework for predicting and optimizing the network performance (Section IV). Finally, we conclude the paper and discuss our future work in Section VI.

\section{EMPIRICAL ANALYSIS OF RSS AND PER}

In this section, we study the statistical properties of signal strength and error rates. We first describe the testbed infrastructure, and then analyze the PER over a broad range of RSS. This analysis is helpful for categorizing different types of links. We then perform distribution-fitting and independence tests for observed RSS and PER values over a single link.

\section{A. Measurement Methodology}

The experiments were conducted using our indoor testbed that consists of six nodes placed in offices along a corridor as shown in Figure 1. Each node consists of a Soekris board [8] with mini-PCMCIA wireless card running Atheros chipset [9] and a modified madwifi driver [10]. The wireless cards operate on 802.11a to avoid interference with the resident $802.11 \mathrm{~b} / \mathrm{g}$ network in the building, and spectrum analyzers were used to ensure absence of external interference. Spurious transmission diveresity of antennas [11] is turned off, and rate-control modules are disabled.

\section{B. Link categories based on RSS and PER}

We use existing classification of links which have categorized the links into three zones based on the observed PER [12]: (1) A Low-loss zone where the links observe very few packet losses; (2) A High-loss zone where the link experiences very high rate of packet errors; and (3) An intermediate Gray-zone where the link quality fluctuates between low and high losses. We use the terms strong, average and weak link to refer to the links in low-loss, gray and highloss zone, respectively. We use these categories to summarize the properties of the links since, as we demonstrate later, links in each category exhibit similar statistical behavior.

Figure 2 shows the Packet Error Rate (PER) against the measured RSS values for different types of links under $6 \mathrm{Mbps}$

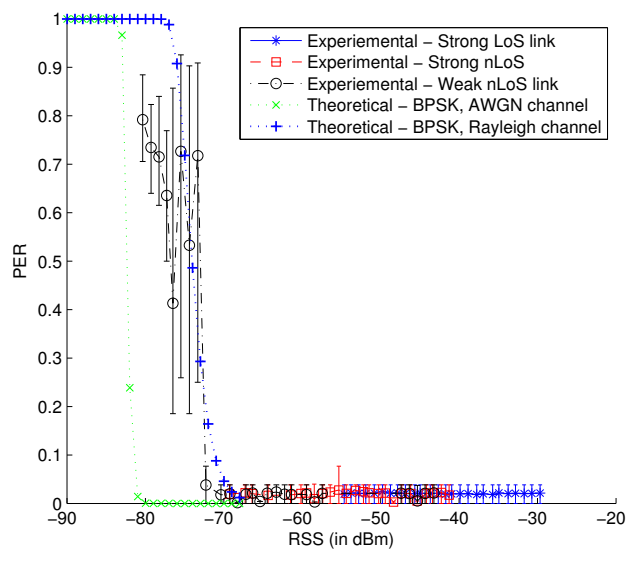

Fig. 2. PER vs. RSS: PER is constant for strong links and has high variance for gray-zone links.

transmission rate. Theoretical BER curves were calculated for BPSK modulation with convolutional codes (as per 802.11a specifications) under AWGN and Rayleigh channels. As observed in other studies [13], theoretical curves do not match the obtained PER. It underestimates the PER of strong links and overestimates the PER of weak links. Hence, theoretical models cannot be directly used for accurate prediction.

The division of PER into three piecewise zones is clearly identified by observing the experimental PER curves, and mean and deviation of each cateogory of link is varying. Strong links have low and constant PER with small variance. PER in gray zone varies widely (from 0.2 to 0.9 ). PER of weak links approaches 1 with acceptable variation. In the gray zone, we observe that aggregate metrics like mean PER is not sufficient to capture the error rate due its huge variation. Section II-D investigates this region in detail.

\section{Distribution and independence of RSS}

The approximation of RSS as a constant or a sequence of i.i.d. random variables from some distribution has been extensively used in the literature [1], [5]. However, the validity of these assumptions has not been extensively tested, even though the i.i.d. assumption has a large effect on the effectiveness of the model. In this section, we analyze the empirical RSS values to infer about the independence and distribution.

Independence and distribution-fitting tests are applied for RSS values collected in 1.5 second intervals. Independence test is conducted by observing the Auto-correlation function (ACF) decay at different lags ${ }^{1}$. We conduct distribution-fitting tests using Kolmogorov-Smirnoff (KS) and Log-likelihood tests (and Lillifor test, if applicable). Distribution fitting tests were carried out for 10 well-known distributions and we present the

\footnotetext{
${ }^{1} \mathrm{ACF}$ is a statistical metric that indicates the correlation between successive points in a time-series data. ACF is defined at various positive integer points $n$ (called as lags) and is a value between $[0,1]$ at each lag. ACF at lag $n$ denotes the correlation between the measurement point at time $t$ and $t+n$. A perfect random variable should have a ACF of 1 for lag 0 and ACF of 0 at all the other lags.
} 
TABLE I

DISTRIBUTION AND INDEPENDENCE TEST FOR RSS: RESULTS OF Normal(N), LOG-NORMAL(LN), Gamma(G) AND Weibull(W) ARE SHOWN. RESULTS OF KS AND INDEPENDENCE TESTS SHOWS THAT WEAK-LINK CAN BE APPROXIMATED AS A I.I.D. RANDOM VARIABLE FROM LOG-NORMAL DISTRIBUTION. KS TEST REJECTS ALL THESE DISTRIBUTIONS FOR STRONG AND AVERAGE LINKS.

\begin{tabular}{|c|c|c|c|c|c|}
\hline \multirow[b]{2}{*}{ Link } & \multicolumn{4}{|c|}{$\begin{array}{l}\text { Distribution test: (\%age } \\
\text { samples not rejected) }\end{array}$} & \multirow{2}{*}{$\begin{array}{c}\text { Independence test } \\
\text { Median ACF } \\
\text { at Lag } 1\end{array}$} \\
\hline & $\mathrm{N}$ & $\mathrm{LN}$ & $\mathrm{G}$ & $\mathrm{W}$ & \\
\hline Strong & 8.9 & 9.7 & 10.4 & 9.0 & -0.0015 \\
\hline Average & 14.2 & 12.7 & 11.9 & 16.4 & 0.014 \\
\hline Weak & 59.0 & 70.9 & 65.8 & 69.3 & -0.029 \\
\hline
\end{tabular}

results for distributions that are commonly used for modeling RSS.

We summarize the results in Table I. Only weak links can be coarsely approximated as log-normal distribution, suggesting that the approach used by Qiu et al. [1] is valid only for weak links. Visual observation of histograms indicate that RSS for the strong link is well-approximated as a constant since there is very low variance (as observed by Reis et al. [2]). Detailed results can be found in the technical report [14].

\section{Determining the cross-over RSS values}

The cross-over points from low-loss to gray zone (henceforth called as cross-over point) varies for different links. Hence, constant cross-over points cannot be assumed for estimating the category of the link. As we have seen in Section II-C, the variance of RSS is small and gradually moves from constant towards log-normal distribution as the RSS decreases. Hence, the cross-over point cannot be concluded from measured RSS values.

Figure 3 shows the histograms of PER and RSS values for link from node 2 to 5 in our testbed. We vary the transmit power at the sender and observe its effect on PER and RSS. The PER is unimodal and low for high-transmit powers (Figure 3(a)) and has a higher values with unimodal distribution for low-transmit powers (Figure 3(c)).

Intuitively, we expect the PER histogram to shift from 0 to 1 (with probably increased variance) as we decrease the transmit power. However, Figure 3(b) shows that at a certain intermediate RSS value, the PER fluctuates between low and high values over a very small RSS range (even on a time scale of fraction of seconds). It becomes bimodal with a large separation between the two modes. A small difference in the signal levels, which may be caused by a fading channel, drastically alters the PER [15]. This indicates that a link should not only be aware of the RSS values, but also the precise nature of the channel between them to quantify the quality of the link. Disseminating or measuring the channel information (e.g. the PER for a large range of RSS values) is valuable when there is a high probability that link operates in this gray-zone.

\section{E. PER distribution}

In this paragraph, we statistically analyze the empirical PER values in all the zones to check if they can be assumed to be
TABLE II

DistRIBUTION AND INDEPENDENCE TEST FOR PER: RESULTS OF NORMAL(N), LOG-NORMAL(LN), GAMma(G), BETA(B) AND WEIBULL(W) ARE SHOWN. WEAK-LINKS CAN BE APPROXIMATED AS I.I.D. RANDOM VARIABLES FROM BETA OR LOG-NORMAL DISTRIBUTION AND AVERAGE LINKS HAVE MEMORY.

\begin{tabular}{|c||c|c|c|c|c||c|}
\hline \multicolumn{1}{|c||}{} & \multicolumn{4}{c||}{ Distribution test: (\%age } & \\
samples not rejected) & Memory? \\
Link & N & LN & G & B & W & \\
\hline Strong & 2 & 1 & 1 & 1 & 1 & - \\
Average & - & - & - & - & - & Yes \\
Weak & 60 & 93 & 94 & 62 & 91 & No \\
\hline
\end{tabular}

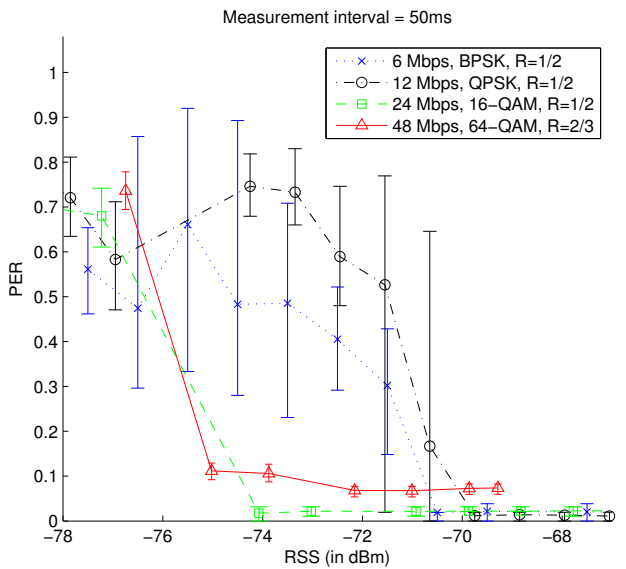

Fig. 4. Effect of transmission rate on PER.

i.i.d. random variables from some distribution. We measure PER once in every $50 \mathrm{~ms}$. Since we are interested in analyzing distribution in one measurement period, we group the PERs in one measurement period (1.5 seconds) and perform distribution fitting tests, similar to Section II-C, on the data and the results are shown in Table II. It was observed that the ACF of the gray-zone link decays slowly, thus indicating strong memory.

In summary, observing the results of independence and the distribution of PER in the time-frame of seconds, we can infer that: (i) PER of strong links should be best approximated as a constant (Figure 3(a)); (ii) PER of gray-zone links are unpredictable, have memory and are bimodally distributed; and (iii) PER of the high-loss links can be approximated by i.i.d. random variable from a Log-normal, Beta or Weibull distributions.

\section{F. Effect of modulation and transmission rate}

In this section, we analyze the results for different transmission rates on a given link. Measurements were carried out for all the standard transmission rates and a representative subset was chosen to illustrate the effect in a simple and uncluttered manner.

Figure 4 studies the effect of transmission rate on the observed PER for a subset of 802.11a transmission rates. 


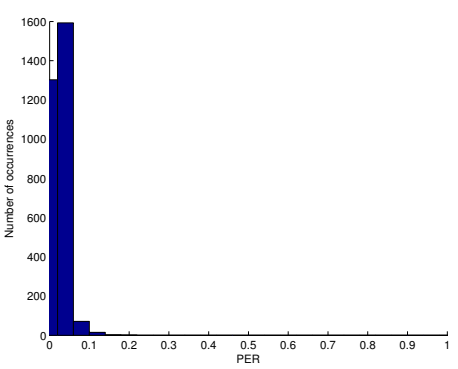

(a) PER at $6 \mathrm{dBm}$ transmit power

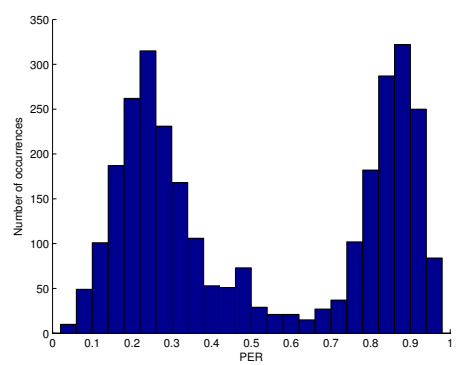

(b) PER at $3 \mathrm{dBm}$ transmit power

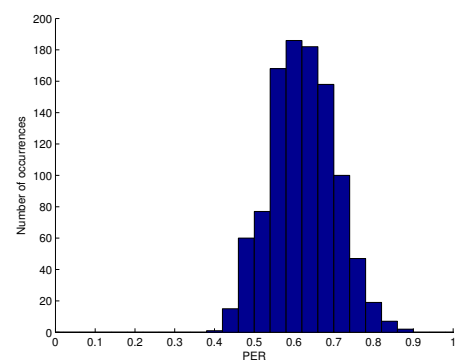

(c) PER at $0 \mathrm{dBm}$ transmit power

Fig. 3. Effect of fading on PER: Histograms of PER is (i) unimodal with very small variance in low-loss zone; (ii) bi-modal and varies widely in gray-zone; and (iii) unimodal with relatively larger variance in high-loss zone.

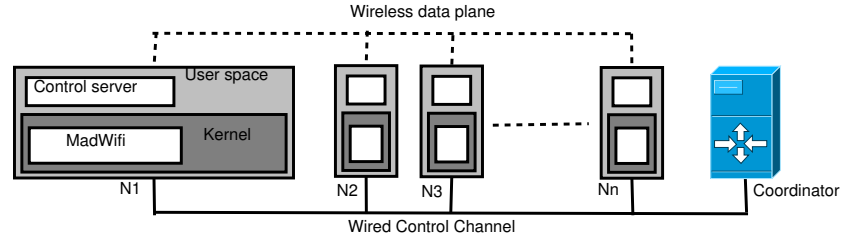

Fig. 5. System diagram of measurement testbed

There is uncertainty in predicting the effect of transmission rate on PER. The common and unintuitive result observed across various traces we examined was the absence of a monotonic trend where PER increased as higher transmission rate is chosen. Also, the cross-over points for some higher transmission rates occur at a lesser RSS than those of lower transmission rates. The above results have serious implications on the rate-control modules since a majority of the modules decrement the transmission rate when there are packet errors, which is shown to be ineffective by our results. The greater performance benefits of rate-control modules which do not always follow this conventional scheme (e.g. [3]) can be explained from our results.

\section{Real-time Measurement Framework}

The analysis in the previous section demonstrate the necessity to measure RSS and PER for links in a wireless network, and described mechanisms to conclude several important parameters such as category of the link and cross-over points. While estimation of these parameters assist higher layer protocols, the process of real-time measurement and estimation poses practical challenges in terms of coordination between the nodes and measurement overheads. In this section, we briefly describe the system components that enable real-time measurement of link quality on our testbed.

\section{A. System architecture}

Figure 5 shows the system architecture of the existing testbed. The wireless nodes are represented by $N_{i}$. The modified Madwifi driver records the information for all the packets where the header is received correctly. Currently, we record the time on chipset (in microseconds), RSSI, noise, sender, receiver, sequence number and error. Recent packet reception information are accessed at the user space through the /proc file system.

At the user space, each wireless node runs a Control Server with a socket open to send and receive control messages and requests. Configuration commands (e.g. set transmission power, rate) and measurement operations (e.g. read received packet information, PER measurement) at the wireless nodes can be remotely executed by issuing commands to the control server. This control server interface enables both distributed and centralized control of wireless nodes.

In the current version, a Central coordinator controls our wireless mesh network to provide real-time link quality.

\section{B. Measurement protocol}

The measurement protocol, which is currently initiated by the coordinator node, performs two basic functionalities: (a) Polling receiver traces and (b) PER measurement.

Polling receiver traces tracks the dynamic changes in the link quality by polling each node for the received packet information over the wired control channel. This process is relatively non-intrusive, simple and light-weight. A remote request to the control server on each node to transmit the packet traces fetches the current trace. For each node, it updates the statistical measures of RSSI (mean, median and variance) from other nodes.

The PER measurement protocol is more complex and intrusive. The PER values vary on different transmission parameters such as transmission power, modulation scheme (transmission rate) used, and packet size. Hence, measuring PER values for each combination of the transmission parameter and each link has a high complexity. We have implemented a lowoverhead PER module where each node broadcasts only once for a set of representative transmission parameters. The rest of the nodes sense and report the observed MAC trace to the coordinator. The coordinator builds the PER table that provides the statistics about the observed RSSI statistics and PER. Since this module has significant control messages, care should be taken to initiate when the data traffic is lesser (e.g. night and weekends). 
The use of broadcast instead of unicast is beneficial for two reasons. First, every node has to broadcast only once instead of unicasting once for each receiver of a link. Hence, it reduces the complexity of the protocol from $O\left(n^{2}\right)$ to $O(n)$, where $n$ is the number of nodes. Second, it avoids the effects of MAC protocols such as retries and exponential back-off. The effect of MAC protocol can be predicted by using the existing MAC models with the measured PER data [16], [17].

While the current implementation of measurement protocol is initiated by the central coordinator, the protocol can be optimized and implemented in a distributed manner using adaptive mechanisms. This is an area of our future work.

\section{Applications of Real-time Measurement}

As we concluded in Section II, theoretical estimation of received signal and PER does not match observed values, and realistic measurement protocols enhance various higher layer modules such as capacity estimation and rate-adaptation. The real-time measurement protocol forms a fundamental entity to enable such higher level applications in wireless systems. We discuss our primary results of two applications that use realtime measurement protocol in our wireless network: Powercontrol and network monitoring tool.

a) Power-control: In Section II-B, we showed that Packet Error Rate (PER) is stable and constant for a strong link (RSS values above the cross-over point). Thus, the performance of a strong link is the same irrespective of how high the RSS value is above the cross-over point. Transmitting at lower power - while ensuring that the RSS is above the cross-over point - decreases the amount of interference that the source causes to the neighboring nodes. This permits higher reuse of channel and increase in network throughput.

As described in Section III-B, the measurement protocol maintains a PER table at the controller. This table maintains the cross-over point (minimum RSS value for strong link) for each link and modulation rate. Transmission on each link is started with default highest transmission power. The RSS at the receiver is constantly reported to the coordinator via the control channel. The control channel adaptively instructs the sender to alter its transmission power based on the difference between the observed RSS and lowest RSS required to be above the cross-over point. Finally, the sender converges to a transmission power that yields lowest RSS for a strong link.

We run this protocol on several different scenarios, where a pair of random links are selected in each scenario. Figure 6 shows change in throughput as the power-control algorithm adapts to the ideal transmit power. We observe that exposed terminals are eliminated (Scenarios 1,2 and 3). In other scenarios, the overall network throughput is unaffected. Out of 13 scenarios, we observed the measurement protocol introduced hidden terminal in just one scenario. This demonstrates that power-adaptation based on PER measurement, in general, leads to greater system throughput.

b) Network monitoring: The periodic results from the measurement protocols are useful to visually monitor the link quality. We have developed a prototype of this tool that

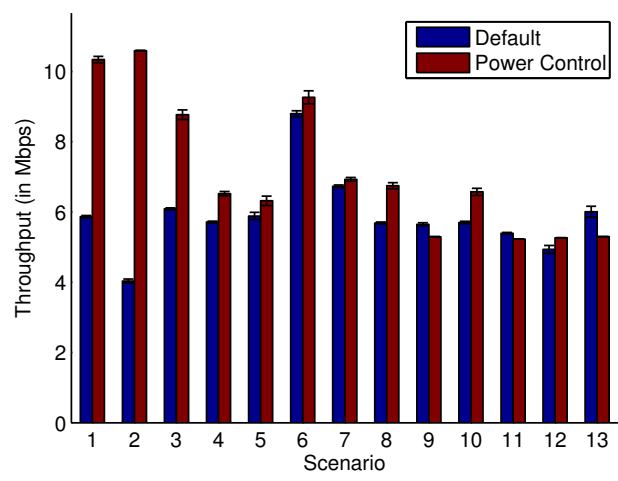

Fig. 6. Dynamic power adaptation

we use to summarize and analyze receiver traces and PER measurement data. This tool plots real time data for link quality graphs like RSSI time-line, PER statistics and their distributions. Sample output of the network monitoring tool is available at our website [18]. Such a tool provides visually intuitive and real-time network quality metrics that enable researchers and network administrators with summary of the vast data collected.

\section{RELATED WORK}

Several empirical studies and MBAs have analyzed the data gathered from IEEE 802.11 wireless networks. For the sake of clarity, we study the related work under the below three categories.

Temporal stability of RSS: Some studies conclude that RSS fluctuates rapidly over fraction of seconds [19], while other studies [2], [20], [21] concur with our observation that the RSS values remains representative in the order of seconds. Unlike the above papers, we measure the stability of RSS in more detail: we show the variation of the RSS for different categories of links and map the stability of the RSS with respect to the empirical error rate and the zone of the link. We believe that this is of direct use in the design of MBAs.

Inference of statistical distribution: A discord about the distribution of the RSS and PER is prevalent seen in the literature. Some studies infer large variations in observed RSS [20] while others observe smaller variations [2], [21]. RSS is verified to be constant in some experiments [2] and lognormally distributed in others [1]. In addition to comparing the RSS to several distributions and performing tests to check the independence, we show that the distribution of RSS can follow constant or log-normal behavior based on the operating zone of the link. Hence, our contribution solves the controversy by unifying conflicting conclusions. We also study the statistical tests to infer the independence and distribution of the PER which is assumed as constant [5], as a log-normal or normal i.i.d. random variable [1], [7]. Guha et al. [13] study the variation of the SNR with time for different links. However, their objective is to quantify the amount of memory for different type of links and they not conclude on the distribution 
of these variables. Further, most of the existing studies [13], [20], [22] use the $2.4 \mathrm{GHz}$ band which is prone to high external interference from Wi-Fi traffic, thus leading to false conculsions about the propagation effects. We avoid this by using the $5 \mathrm{GHz}$ band and ensure the absence of external interference by using spectrum analyzers.

Effect of fading and gray zone: Existing studies [2], [12] have categorized links into high-loss, low-loss and gray zones. However, they have not analyzed the unifying statistical properties for these zones. The bimodal distribution of PER due to the effect of fading is observed by some existing studies [12], [15]. However, the study in [15] is simulation based and Zuniga et al. [12] measure using sensor motes which does not use IEEE 802.11 protocol.

\section{CONCLUSION AND FUTURE WORK}

This paper presented an empirical analysis of the RSS and error rates by focusing on the requirements for the measurement-based applications deployed in a IEEE 802.11 based networks. We contribute to the existing measurement based research by analyzing the temporal properties, independence and the distribution of received signal strength and error rate. We concluded these statistical properties vary with the zone in which the link operates and are similar for links belonging to the same category. We also observed the bi-modal distribution of the PER in the gray zone links, which is as a result of fading. Our PER results showed the importance of identifying the transitions between a strong and a weak link and we sketched the mechanisms to identify such a transition. The effect of transmission-rates on PER is non-monotonic and the trend is not predictable with the data available from the wireless cards. We demonstrated a real-time measurement protocol, and discussed example applications that can utilize measured RSS and PER values.

In future, we plan to perform more detailed experiments with software defined radios which are capable of providing the data at a higher granularity. Detecting and accounting for the external interference by using the measured values is a part of our future work. The long-term goal of this line of work is to construct a realistic, low-overhead and accurate measurement mechanisms that can be used for network planning, provisioning and optimization of higher layer protocols.

\section{REFERENCES}

[1] L. Qiu, Y. Zhang, F. Wang, M. K. Han, and R. Mahajan, "A general model of wireless interference," in MobiCom '07: Proceedings of the 13th annual ACM international conference on Mobile computing and networking. New York, NY, USA: ACM, 2007, pp. 171-182.

[2] C. Reis, R. Mahajan, M. Rodrig, D. Wetherall, and J. Zahorjan, "Measurement-based models of delivery and interference in static wireless networks," SIGCOMM Comput. Commun. Rev., vol. 36, no. 4, pp. 51-62, 2006.

[3] S. H. Y. Wong, H. Yang, S. Lu, and V. Bharghavan, "Robust rate adaptation for 802.11 wireless networks," in MobiCom '06: Proceedings of the 12th annual international conference on Mobile computing and networking. New York, NY, USA: ACM, 2006, pp. 146-157.

[4] R. Draves, J. Padhye, and B. Zill, "Comparison of routing metrics for static multi-hop wireless networks," in SIGCOMM, 2004.
[5] D. Lal, A. Manjeshwar, F. Herrmann, E. Uysal-Biyikoglu, and A. Keshavarzian, "Measurement and characterization of link quality metrics in energy constrained wireless sensor networks," Global Telecommunications Conference, 2003. GLOBECOM '03. IEEE, vol. 1, pp. 446-452 Vol.1, Dec. 2003.

[6] K. Jain, J. Padhye, V. N. Padmanabhan, and L. Qiu, "Impact of interference on multi-hop wireless network performance," in MobiCom, 2003.

[7] A. Woo, T. Tong, and D. Culler, "Taming the underlying challenges of reliable multihop routing in sensor networks," in SenSys '03: Proceedings of the 1st international conference on Embedded networked sensor systems. New York, NY, USA: ACM Press, 2003, pp. 14-27.

[8] "Soekris4826." [Online]. Available: http://www.soekris.com/net4826. $\mathrm{htm}$

[9] “Atheros/AR5006chipset." [Online]. Available: http://www.atheros.com/ pt/AR5006Bulletins.htm

[10] "Madwifi: Linux driver for atheros cards," http://madwifi.org/.

[11] D. Giustiniano, I. Tinnirello, L. Scalia, and A. Levanti, "Revealing transmit diversity mechanisms and their side-effects in commercial ieee 802.11 cards," Telecommunication Networking Workshop on QoS in Multiservice IP Networks, 2008. IT-NEWS 2008. 4th International, pp. 135-141, Feb. 2008.

[12] M. Zuniga and B. Krishnamachari, "Analyzing the transitional region in low power wireless links," Sensor and Ad Hoc Communications and Networks, 2004. IEEE SECON 2004. 2004 First Annual IEEE Communications Society Conference on, pp. 517-526, Oct. 2004.

[13] R. K. Guha and S. Sarkar, "Characterizing Temporal SNR Variation in 802.11 Networks," Vehicular Technology, IEEE Transactions on, vol. 57, no. 4, pp. 2002-2013, July 2008.

[14] V. Kolar, S. Razak, P. Mahonen, and N. Abu-Ghazaleh, "Empirical validation of the modeling assumptions about signal strengh and error rates under a fading wireless channel," Dept. of Computer Science, SUNY, Binghamton, Tech. Rep. R-09-02 (http://www.cs.binghamton. edu/\%7Evinkolar/pubs/kolarR0902.pdf), Dec. 2009.

[15] O. Awoniyi and F. Tobagi, "Effect of fading on the performance of voip in ieee 802.11 a wlans," Communications, 2004 IEEE International Conference on, vol. 6, pp. 3712-3717 Vol.6, June 2004.

[16] S. Razak, N. Abu-Ghazaleh, and V. Kolar, "Modeling of two-flow interactions under sinr model in multi-hop wireless networks," in 33rd IEEE Conference on Local Computer Networks(LCN) 2008., Oct., pp. 297-304.

[17] V. Kolar, K. Bharath, N. B. Abu-Ghazaleh, and J. Riihijarvi, "Contention in multi-hop wireless networks: model and fairness analysis," in MSWiM '09: Proceedings of the 12th ACM international conference on Modeling, analysis and simulation of wireless and mobile systems. New York, NY, USA: ACM, 2009, pp. 21-29.

[18] "Network Monitoring Framework in Wireless Networks." [Online]. Available: \url\{http://www.cs.binghamton.edu/\%7Evinkolar/research/ netmon/\}

[19] A. Keshavarzian, E. Uysal-Biyikoglu, D. Lal, and K. Chintalapudi, "From experience with indoor wireless networks: A link quality metric that captures channel memory," Communications Letters, IEEE, vol. 11, no. 9, pp. 729-731, September 2007.

[20] V. Shrivastava, D. Agrawal, A. Mishra, S. Banerjee, and T. Nadeem, "Understanding the limitations of transmit power control for indoor wlans," in IMC '07: Proceedings of the 7th ACM SIGCOMM conference on Internet measurement. New York, NY, USA: ACM, 2007, pp. 351364.

[21] K. Srinivasan and P. Levis, "RSSI is Under Appreciated," Third Workshop on Embedded Networked Sensors (EmNets 2006), May 2006.

[22] D. Aguayo, J. Bicket, S. Biswas, G. Judd, and R. Morris, "Linklevel measurements from an $802.11 \mathrm{~b}$ mesh network," in SIGCOMM '04: Proceedings of the 2004 conference on Applications, technologies, architectures, and protocols for computer communications. New York, NY, USA: ACM Press, 2004, pp. 121-132. 